\title{
Quantifying the Pressure-dependence of Work of Adhesion in Silicon-Diamond Contacts
}

\author{
Rimei Chen ${ }^{1 *}$, Sai Bharadwaj Vishnubhotla ${ }^{2 *}$, Subarna R. Khanal, ${ }^{2}$ Tevis D. B. Jacobs ${ }^{2, a)}$, and Ashlie \\ Martini ${ }^{1, a)}$ \\ ${ }^{1}$ Department of Mechanical Engineering, University of California-Merced, 5200 North Lake Road, \\ Merced, CA, 95343, USA \\ ${ }^{2}$ Department of Mechanical Engineering and Materials Science, University of Pittsburgh, 3700 O'Hara \\ St., Pittsburgh, PA, 15261, USA \\ * indicates equal contribution \\ a) Authors to whom correspondence should be addressed: amartini@ucmerced.edu
}

The published version of this article can be found at: https://doi.org/10.1063/1.5127533 Full citation information is as follows:

Chen, R., Vishnubhotla, S.B., Khanal, S.R., Jacobs, T.D. and Martini, A.: Quantifying the

Pressure-dependence of Work of Adhesion in Silicon-diamond Contacts. Applied Physics Letters, 116, 051602 (2020).

\begin{abstract}
Continuum mechanics models for contacting surfaces assume a constant interfacial energy, or work of adhesion, between materials. Recent studies have challenged this assumption, instead demonstrating that stress-dependent chemical reactions across the interface modify the work of adhesion. Here, we perform 77 adhesion tests on diamond-silicon contacts using in situ TEM and atomistic simulations to quantify how the adhesion changes as a function of applied pressure. The results show a 7-fold increase in work of adhesion (from approximately 1 to $7 \mathrm{~J} / \mathrm{m}^{2}$ ) with an increase in mean applied pressure from 0 to $11 \mathrm{GPa}$, where the most significant increase occurs above $5 \mathrm{GPa}$. We rule out alternative explanations for the changing work of adhesion, such as electron-beam artifacts, bulk shape change by inelastic deformation, and time-dependent processes such as creep. Therefore, these results confirm the presence of stress-driven chemical reactions in the contact and quantify the resulting change in adhesion of these materials with applied pressure.
\end{abstract}

Keywords: Work of adhesion, Nanocontacts, In situ TEM, Molecular dynamics simulation 
Adhesion is governed by atomic-scale interactions between surfaces, including van der Waals attraction, electrostatic interactions, and covalent bonding. These effects are typically captured by the work of adhesion $W_{a d h}$, the energy required per unit area to separate two flat surfaces. ${ }^{1}$ This property is fundamental to mechanics models that describe the contact between bodies. ${ }^{2}$ Models based on adhesive spheres, ${ }^{3-5}$ and other, more-complex geometries ${ }^{6-8}$ are widely used to predict surface properties of nanoscale contacts in scientific investigations and technological applications. ${ }^{9-11}$

The assumption underlying these models is that the work of adhesion between the surfaces of the bodies remains constant. Recent work has suggested that this may not be the case and, instead, work of adhesion may depend on the loading conditions. Milne et al. ${ }^{12}$ conducted nanoscale sliding and pull-off experiments to show that work of adhesion increased with stress and with speed of sliding prior to pull-off. In particular, it was shown that the measured work of adhesion is a result of a constant contribution due to van der Waals interactions, a term due to interfacial covalent bonding, and an extra term which increases with stress and the smoothness of the sliding probe. Complementary molecular dynamics simulations using silicon probes and diamond substrates revealed that adhesion is impacted by plastic rearrangements in the probe during sliding, i.e., smoothing, and by the presence of probe-substrate covalent bonds at the time of probe pullback. ${ }^{12}$ Furthermore, the simulations showed that hydrogen termination of the diamond, applied load, sliding distance, and initial probe roughness all impact the simulated adhesion. Another recent study ${ }^{13}$ examined the contact size of a silicon probe on diamond during loading and unloading and showed how the loading data could only be fit using a value of work of adhesion that increased with load up to the maximum force that was applied. Here, we present an extensive experiment and simulation study to characterize the variation of work of adhesion with applied pressure for nanoscale silicon/diamond contacts. We conducted 77 single-asperity adhesion tests at varying loads using nanoscale silicon probes against flat diamond surfaces. Tests were performed using both in situ transmission electron microscopy (TEM) and molecular dynamics (MD) simulation.

Experimental adhesion tests were conducted between silicon nanoscale probes and diamond substrates using an in situ nanoindenter inside of a transmission electron microscope operated at $200 \mathrm{keV}$ (see supplementary material S1). A schematic of the setup, which is identical to Ref. 13, is shown in Fig. 1a. The applied forces were measured in the following ways. For forces larger than $200 \mathrm{nN}$, the nanoindenter's 3-plate-capacitor load cell was used for direct measurement of force-time curves (Fig. $1 \mathrm{~b}$ and supplementary video 2). For forces smaller than $200 \mathrm{nN}$, the instantaneous deflection of the cantilever $x_{\text {disp }}$ was determined from the in situ TEM test videos and was multiplied by the pre-calibrated ${ }^{14}$ spring constant $k$ to determine the instantaneous applied forces $F=k x_{\text {disp }}$ (Fig. 1c and supplementary video 1 ). The tests were conducted at a loading/unloading rate of approximately $1 \mathrm{~nm} / \mathrm{s}$. In all tests, the motion of the silicon probe was along the loading/unloading direction and no bending or rolling of the probe apex was observed (see Fig. S1). The mean applied pressure $p_{m}$ at the maximum applied force $F_{\max }$ for a given probe of radius $R$ was calculated using an elastic-plastic mode ${ }^{15}$ for the contact of spheres. In this, the standard, elastic Hertz model is used up to the point where local yielding is predicted; beyond this point, an empirically derived relationship is used to predict contact pressure (see supplementary material S2). The maximum applied forces in the experiments ranged between $0-800 \mathrm{nN}$, corresponding to mean applied pressures ranging from 0 to $11 \mathrm{GPa}$.

Molecular dynamics simulations were used to model nanocontacts of the same material system as the experiments. A schematic of the atomistic model is shown in Fig. 1d and a representative force-time curve is shown in Fig. 1e. A parabolic silicon probe was created with a radius of $3 \mathrm{~nm}$ and was fully terminated with hydrogen. Previous work on nanoscale diamond contacts showed that the work of adhesion depends on the hydrogen coverage. ${ }^{12,16-18}$ Because the termination of the experimental surfaces was unknown, the flat diamond substrate was investigated at hydrogen coverages ranging from 0 to $100 \%$ (see supplementary material S3). A coverage of $85 \%$ was chosen for this study to best match the experimental results in terms 
of the magnitude of the work of adhesion. A Nosé-Hoover thermostat was applied to control the temperature of the system at $300 \mathrm{~K}$. All interactions were modeled by the ReaxFF potential ${ }^{19}$ with the parameter set from Ref. 20 using a time step of 0.25 fs. The normal force was calculated as the sum of the forces on the probe atoms. ${ }^{21}$ The probe was moved towards the substrate at $5 \mathrm{~m} / \mathrm{s}$ until it reached the desired force, was held at this position until the energy reached steady state, and then was retracted from the substrate at 5 $\mathrm{m} / \mathrm{s}$. The simulation loading/unloading speed was much faster than that in the experiments due to the small time-step required by the atomistic simulation method. The maximum forces in the simulation ranged from 3 to $150 \mathrm{nN}$, resulting in a range of mean applied pressures from 3 to $11 \mathrm{GPa}$, consistent with the applied pressures in the experiments. Simulations were carried out using the large-scale atomic/molecular massively parallel simulator (LAMMPS). ${ }^{22}$

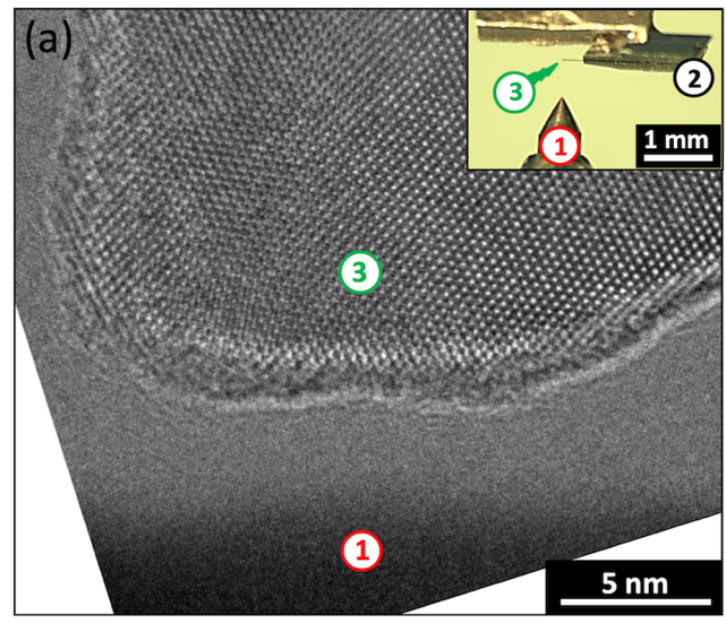

(d)

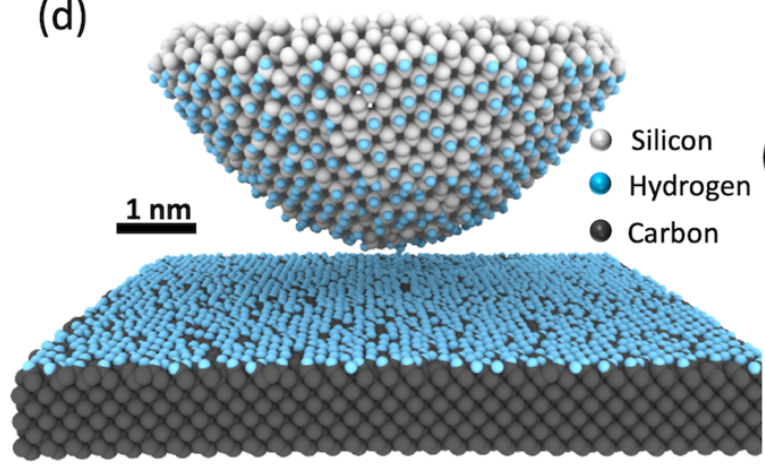

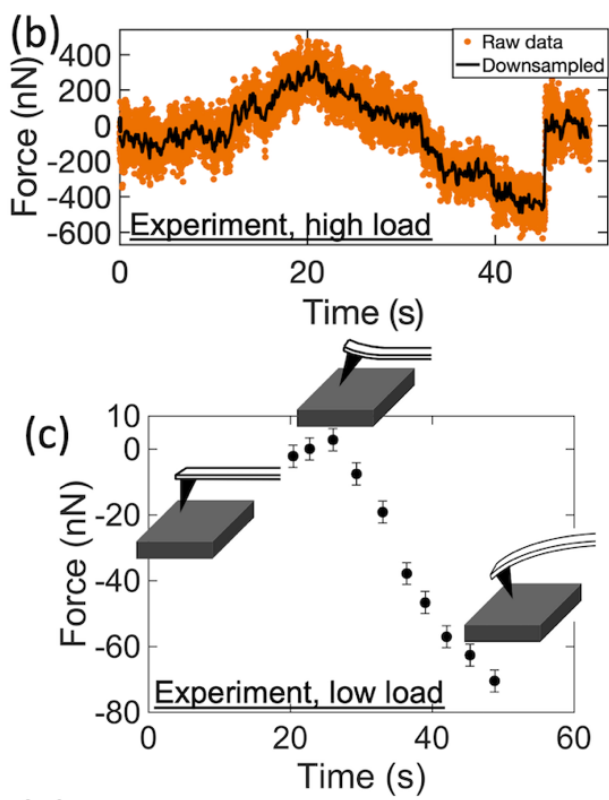

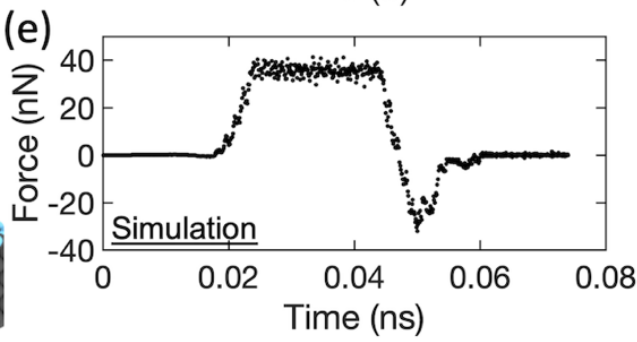

FIG. 1: The adhesion tests were performed using in situ TEM experiments and atomistic simulations. In situ TEM adhesion tests (a) were performed between a flat nanoindenter (1, inset) and an AFM probe (2,3, inset). Forces were measured using either the nanoindenter's load sensor (b) or the deflection of the calibrated cantilever (c). Molecular dynamics simulations with a paraboloidal silicon probe and a flat diamond substrate (d), were used to measure force during loading and pull-off (e).

The work of adhesion $W_{\text {adh }}$ was computed using the Maugis-Dugdale model of adhesive contact from the adhesive force $F_{a d h}$ as: ${ }^{23}$

$$
W_{a d h}=\frac{F_{a d h}}{\chi \pi R}
$$

where $\chi$ is a dimensionless parameter ranging from 1.5 to 2 . The adhesive force $F_{\text {adh }}$ was the maximum adhesive force during retraction, measured from the force-time data in the experiment and simulation (Fig. 1). The radius $R$ of the apex of the probe was measured before and after the test by tracing the outer contour of the probe from the high-resolution TEM image or side view of the atom positions in the 
simulation, and the extracted profile was fit with a parabola of the form $z=x^{2} / 2 R$, where $z$ is the vertical height and $x$ is the lateral dimension.

The value of $\chi$ in Eq. 1 depends on the nature of contact. Its limits correspond to the Derjaguin-MüllerToporov (DMT) model ${ }^{4}(\chi=2)$, which assumes weaker and longer-range adhesive interactions and is applicable for stiffer materials, and the Johnson-Kendall-Roberts (JKR) model ${ }^{3}(\chi=1.5)$, which assumes short-range adhesion and is applicable for softer materials. To determine its value, we used the model of Maugis $^{5}$ and the numerical method of Carpick-Ogletree-Salmeron (COS). ${ }^{23}$ The Maugis parameter ${ }^{5}$ was calculated as:

$$
\lambda=2 \sigma_{0}\left(\frac{9 R}{16 \pi E_{\text {eff }}^{2} W_{\text {adh }}}\right)^{1 / 3}
$$

where $\sigma_{0}$ is the theoretical cohesive stress and $z_{0}$ is the equilibrium separation between the materials. The cohesive stress $\sigma_{0}$ was determined by equating it to the minimum adhesive stress of the Lennard-Jones potential, which gives $\sigma_{0}=W_{a d h} / 0.97 z_{0} \cdot{ }^{23}$ For a silicon-diamond contact, the equilibrium separation $z_{0}$ is $0.25 \mathrm{~nm}^{24-28}$ and the effective modulus $E_{\text {eff }}$ is $124.5 \mathrm{GPa}$, calculated from the elastic modulus of [ $\left.\begin{array}{lll}1 & 0 & 0\end{array}\right]$ diamond $(\mathrm{E}=1050.0 \mathrm{GPa}, v=0.1)^{29}$ and $\left[\begin{array}{lll}1 & 0 & 0\end{array}\right]$ silicon $(\mathrm{E}=130 \mathrm{GPa}, v=0.28) .{ }^{30}$ Using these values of $z_{0}$ and $E_{\text {eff }}$, and the vales of $F_{a d h}$ and $R$ measured in the experiments and simulations, the COS method was applied to simultaneously measure the Maugis parameter $\lambda$ and work of adhesion $W_{\text {adh }}$ for all the tests. It was observed that the Maugis parameter $\lambda$ lies in the range of $0.23-1.56$, thus representing an intermediate case between the DMT and JKR limits. All reported work of adhesion values were subsequently determined based on this implementation of the Maugis-Dugdale model.

The results from 77 adhesion tests are shown in Fig. 2. Together, the experimental and simulation data show an increase in work of adhesion from approximately $1 \mathrm{~J} / \mathrm{m}^{2}$ at zero applied pressure up to $6-8 \mathrm{~J} / \mathrm{m}^{2}$ at high pressures. The increase is gradual at low pressures where deformation is expected to be elastic and then increases rapidly at the higher pressures of elastic-plastic deformation. The results at zero applied pressure agree well with those of Ref. 12, in which sliding and pull-off experiments were conducted under the action of adhesive stress only. Simulations performed in that study with Si probes and diamond surfaces showed that larger loads increased smoothing of the probe and interfacial covalent-bond formation, both of which increase adhesion. The present results show that the work of adhesion continuously increases with applied pressure up to very high values. Simulated testing with other probe sizes and other hydrogen coverages (Fig. S2) show that, while the absolute values of work of adhesion vary between conditions, the trends of increasing work of adhesion with applied pressure are consistent across all model contacts. 


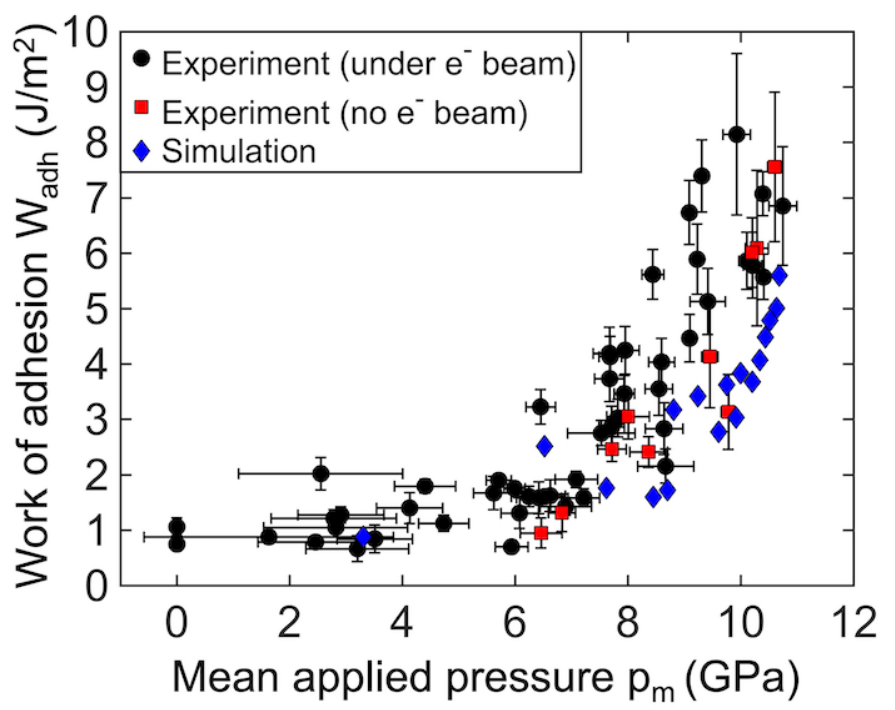

FIG. 2: The measured work of adhesion from experiments and simulations increases approximately seven-fold with applied pressure, where the most significant increase occurs above 5 GPa. The work of adhesion is calculated using the Maugis-Dugdale model; the mean applied pressure is calculated using an elastic-plastic model of contact (see main text). Experimental tests were performed with direct TEM observation (black symbols) and also with the beam off (red symbols), in order to rule out electron-beam artifacts.

These results demonstrate a significant increase in work of adhesion with applied pressure. To support that these changes arise from atomic bonding, rather than other physical origins, we sought to rule out three other common explanations for varying adhesion, including: electron-beam induced reactions; ${ }^{31}$ shape change by inelastic deformation ${ }^{32}$ (such as fracture or gross plastic flow); and time-dependent deformation $^{33-35}$ (such as viscoelasticity or creep). First, to investigate the effect of the electron beam, TEM experiments were conducted with the electron beam switched off (red markers in Fig. 2). The measured work of adhesion still increased with applied pressure, agreeing with the beam-on measurements (black markers in Fig. 2) within the experimental uncertainty. Further evidence that the electron beam did not have a strong effect is provided by post-hoc analysis of beam current during beam-on testing, which demonstrated that deviations in electron dose rates during testing (over the range of 96 to $317 \mathrm{e}^{-} / \mathrm{A}^{2} \mathrm{~s}$ ) had no systematic effect on adhesion results. Consistent with prior results showing that silicon probes are robust to electron beam exposure, ${ }^{36}$ the present experiments showed no evidence of contamination or damage of the probe due to the electron beam.

Second, while large-scale shape change from inelastic deformation (fracture or gross plastic flow) could potentially lead to an increase in contact size and therefore adhesive force, this was ruled out by examining the probes before and after testing. From side-view images of the probes, the exterior profile was extracted and a parabola was fit to the probe's apex (see Fig. 3a and supplementary material S4). For all cases, the average change in radius of the probe apex was just $1 \%$, with no single radius deviating by more than $9 \%$ from the pre-test value. To assess any smaller-scale changes, the best-fit parabola was subtracted from the measured profile leaving only the sub-nm-scale roughness. In all cases, the roughness was approximately equivalent before and after testing: the average change in root-mean-square (RMS) roughness of all the probes was $1 \%$, with no single value of RMS roughness deviating by more than $9 \%$ from the pre-test value. Furthermore, high-resolution images of the probes taken immediately after testing (Fig. 3b and Fig. S3) showed no evidence of dislocations or other defects in the crystal lattice. A similar analysis performed on the simulated probes by tracking atom positions showed no evidence of a change in probe shape (Fig. 3c) or crystal defects (Fig. 3d). Finally, the order of the tests was varied, including repeating multiple tests to the same load and conducting a lower-load test following a higher-load test (Fig. S4). It is expected that 
inelastic material changes will occur when the probe is subjected to a previously unachieved load, but would be less significant upon subsequent testing to a similar or lower load. By contrast, the results (see supplementary material S5) showed that the order of testing had no effect on the measured adhesive force.

(a)
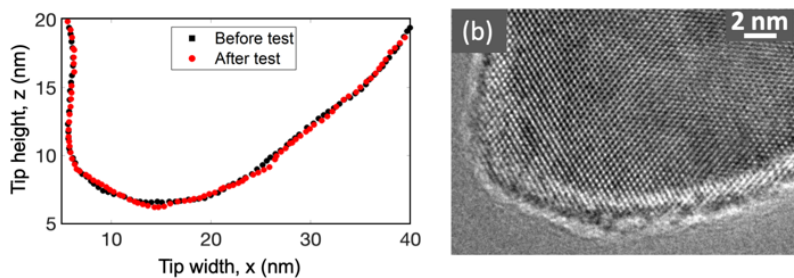

(c)
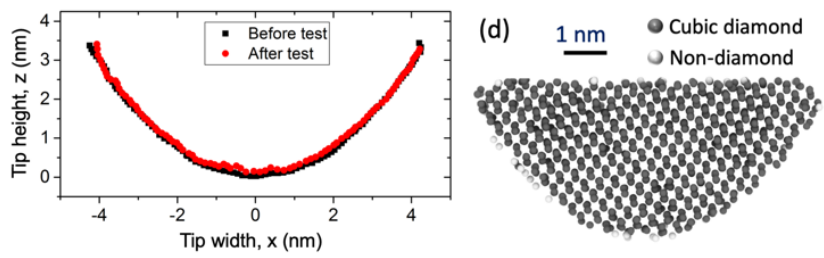

FIG. 3: The increase in work of adhesion with applied pressure is not due to a change in probe shape due to shape inelastic deformation. Comparison of the probe shapes before and after the test suggest no significant shape change in both the experiments (a) and simulations (c). TEM images of the probes (b) and crystallographic analysis of the model (d) after the test showed no evidence of defects in the silicon lattice. See Fig. S3 for further analysis of other representative probes.

Third, to rule out time-dependent phenomena such as creep or viscoelasticity, the adhesion tests were repeated with variation in hold time and pull-off rate. The experimental tests were conducted for five different hold times between 0 to $60 \mathrm{~s}$ at a maximum force of $417 \pm 40 \mathrm{nN}$ with a probe radius of $21.9 \pm$ $1.5 \mathrm{~nm}$. Similarly, simulation tests were conducted for different hold times from 2 to $12 \mathrm{~ns}$ at three maximum forces of $132.2 \pm 2.0,21.8 \pm 0.8$, and $2.2 \pm 0.4 \mathrm{nN}$. The adhesive force showed no significant trends with hold time in both experiments (Fig. 4a) and simulations (Fig. 4b). In experiments on a different probe (radius $41.0 \pm 7.2 \mathrm{~nm}$ ), three contact tests were conducted with an applied maximum force of $197 \pm$ $34 \mathrm{nN}$ and pull-off rate of $0.5,1$, and $2 \mathrm{~nm} / \mathrm{s}$. The pull-off force did not show any significant change with unloading rate in the range of 0 to $2 \mathrm{~nm} / \mathrm{s}$ (Fig. 4c). In the simulations, the rate of pull-off was varied from 2 to $10 \mathrm{~m} / \mathrm{s}$ and there was no notable change of the adhesive force (Fig. 4d). Therefore, the tested range of hold times and speeds had no effect on measured adhesion and cannot explain the increase of work of adhesion with applied pressure. 

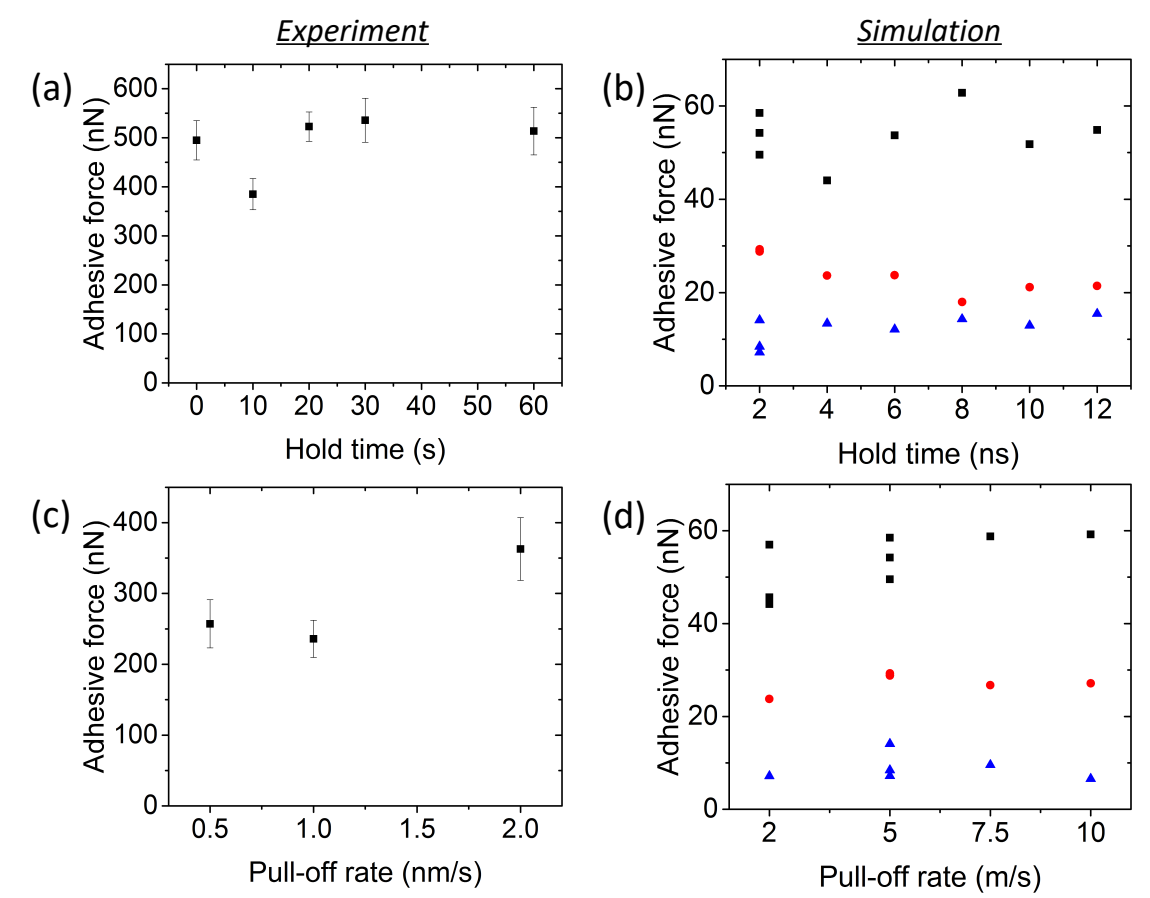

Fig. 4: The increase in work of adhesion with applied pressure cannot be attributed to time-dependent phenomena such as creep or viscoelasticity. In both experiments $(a, c)$ and simulations $(b, d)$, the adhesive force shows no dependence on hold time nor on pull-off rate within the limits explored. In (b) and (d), the black squares, red circles, and blue triangles refer to maximum applied forces of $132 \mathrm{nN}, 21.8 \mathrm{nN}$, and $2.2 \mathrm{nN}$, respectively, for a probe radius of $3 \mathrm{~nm}$. Although the timescales of the experiment and simulation differ significantly, these plots confirm that the adhesive force does not depend on the hold time or pull-off rate within the range of times and rates accessible to each method.

Since the possible effects of three common explanations for varying adhesion-electron-beam induced reactions, shape change by inelastic deformation, and time-dependent deformation-were excluded, the present results are attributed to chemical bonding across the interface that is facilitated by stress as suggested by prior experiments and MD simulations; ${ }^{12,13}$ we show that these trends continue and are accelerated with applied pressure. Specifically, the increase in measured work of adhesion corresponds to a stress-driven increase in interfacial bond density, requiring a larger energy per unit area to separate the surfaces. TEM images before and after contact were compared and no material transfer was identified within the detection limits of the instrument $(0.2 \mathrm{~nm}$ on the probe based on the TEM resolution and $1 \mathrm{~nm}$ on the diamond due to vibration of the indenter). Additionally, there was not more than a few atoms of material transfer in the simulated testing. Therefore, while the bond density appears to increase with applied load, the separation of the bodies still occurs at the original interface between the materials.

In summary, this study quantified the increase of the work of adhesion between silicon and diamond with externally applied pressure. Using 77 compression-and-adhesion tests performed on well-controlled silicon-diamond interfaces inside a TEM and complementary atomistic simulations, we found that the strength of adhesion increases with applied pressure. After systematically ruling out other explanations for varying work of adhesion, the increase is attributed to changes in atomic bonding across the interface. This effect causes a seven-fold increase in adhesion with externally applied pressures up to $11 \mathrm{GPa}$. In general, the findings reported here support newer models of contact, in which the work of adhesion is not represented as a static property of the interface, but instead as having a well-defined functional dependence on applied pressure. 


\section{Supplementary Material}

See Supplementary Material for further experimental details; calculation of the mean applied pressure; trends in simulated-probe results with hydrogen termination and probe size; determination of experimental probe radius, shape, and crystal structure; and effect of test order in the experiments; as well as two videos of in situ TEM experiments at low load and high load.

\section{Acknowledgments}

The authors acknowledge funding from the National Science Foundation under awards CMMI-1536800 and CMMI-1537613. The authors acknowledge the use of the Nanoscale Fabrication \& Characterization Facility in the Petersen Institute of NanoScience and Engineering at the University of Pittsburgh. 


\section{References}

${ }^{1}$ J.N. Israelachvili, Intermolecular and Surface Forces: Revised Third Edition (Academic press, 2011).

${ }^{2}$ T.D.B. Jacobs and A. Martini, Appl. Mech. Rev. 69, 060802 (2017).

${ }^{3}$ K.L. Johnson, K. Kendall, and A.D. Roberts, Proc. R. Soc. London A Math. Phys. Eng. Sci. 324, 301 (1971).

${ }^{4}$ B. V Derjaguin, V.M. Muller, and Y.P. Toporov, J. Colloid Interface Sci. 53, 314 (1975).

${ }^{5}$ D. Maugis, J. Colloid Interface Sci. 150, 243 (1992).

${ }^{6}$ H. Gao and H. Yao, Proc. Natl. Acad. Sci. U. S. A. 101, 7851 (2004).

${ }^{7}$ E.D. Reedy, J. Mater. Res. 21, 2660 (2006).

${ }^{8}$ D.S. Grierson, J. Liu, R.W. Carpick, and K.T. Turner, J. Mech. Phys. Solids 61, 597 (2013).

${ }^{9}$ A.M. Minor, E.T. Lilleodden, M. Jin, E.A. Stach, D.C. Chrzan, and J.W. Morris, Philos. Mag. 85, 323 (2005).

${ }^{10}$ W.N. Unertl, J. Vac. Sci. Technol. A Vacuum, Surfaces, Film. 17, 1779 (1999).

${ }^{11}$ H.D. Espinosa, R.A. Bernal, and M. Minary-Jolandan, Adv. Mater. 24, 4656 (2012).

${ }^{12}$ Z.B. Milne, J.D. Schall, T.D.B. Jacobs, J.A. Harrison, and R.W. Carpick, ACS Appl. Mater. Interfaces 11, 40734 (2019).

${ }^{13}$ S.B. Vishnubhotla, R. Chen, S. Khanal, X. Hu, A. Martini, and T.D.B. Jacobs, Tribol. Lett. 67, 97 (2019).

${ }^{14}$ J. E. Sader, J. W. M. Chon, and P. Mulvaney, Rev. Sci. Instrum. 70, 3967 (1999).

${ }^{15}$ L. Kogut and I. Etsion, J. Appl. Mech. 69, 657 (2002).

${ }^{16}$ R.A. Bernal, P. Chen, J.D. Schall, J.A. Harrison, Y.-R. Jeng, and R.W. Carpick, Carbon N. Y. 128, 267 (2018).

${ }^{17}$ A. V. Sumant, D.S. Grierson, J.E. Gerbi, J.A. Carlisle, O. Auciello, and R.W. Carpick, Phys. Rev. B 76, 235429 (2007).

${ }^{18}$ P.L. Piotrowski, R.J. Cannara, G. Gao, J.J. Urban, R.W. Carpick, and J.A. Harrison, J. Adhes. Sci. Technol. 24, 2471 (2010).

${ }^{19}$ A. C. T van Duin, S. Dasgupta, F. Lorant, and W. A. Goddard, J. Phys. Chem A. 105, 9396 (2001).

${ }^{20}$ J. Yeon and A. C. T. Van Duin, J. Phys. Chem. C 120, 305 (2016).

${ }^{21}$ K.E. Ryan, P.L. Keating, T.D.B. Jacobs, D.S. Grierson, K.T. Turner, R.W. Carpick, and J.A. Harrison, Langmuir 30, 2028 (2014).

${ }^{22}$ S. Plimpton, J. Comput. Phys. 117, 1 (1995).

${ }^{23}$ R.W. Carpick, D.F. Ogletree, and M. Salmeron, J. Colloid Interface Sci. 211, 395 (1999).

${ }^{24}$ M. Enachescu, R.J.A. van den Oetelaar, R.W. Carpick, D.F. Ogletree, C.F.J. Flipse, and M. Salmeron, Phys. Rev. Lett. 81, 1877 (1998).

${ }^{25}$ J. Katainen, M. Paajanen, E. Ahtola, V. Pore, and J. Lahtinen, J. Colloid Interface Sci. 304, 524 (2006).

${ }^{26}$ D.L. Liu, J. Martin, and N.A. Burnham, Appl. Phys. Lett. 91, 43107 (2007).

${ }^{27}$ D.S. Grierson, E.E. Flater, and R.W. Carpick, J. Adhes. Sci. Technol. 19, 291 (2005).

${ }^{28}$ T.D.B. Jacobs, J.A. Lefever, and R.W. Carpick, Adv. Mater. Interfaces 2, 1400547 (2015).

${ }^{29}$ H.J. McSkimin and P. Andreatch Jr, J. Appl. Phys. 43, 2944 (1972).

${ }^{30}$ M.A. Hopcroft, W.D. Nix, and T.W. Kenny, J. Microelectromechanical Syst. 19, 229 (2010).

${ }^{31}$ K. Zheng, C. Wang, Y.-Q. Cheng, Y. Yue, X. Han, Z. Zhang, Z. Shan, S.X. Mao, M. Ye, Y. Yin, and E. Ma, Nat. Commun. 1, 24 (2010).

${ }^{32}$ R.G. Horn, J.N. Israelachvili, and F. Pribac, J. Colloid Interface Sci. 115, 480 (1987).

${ }^{33}$ Y.L. Chen, C.A. Helm, and J.N. Israelachvili, J. Phys. Chem. 95, 10736 (1991).

${ }^{34}$ M.P. de Boer, J.A. Knapp, T.A. Michalske, U. Srinivasan, and R. Maboudian, Acta Mater. 48, 4531 (2000).

${ }^{35}$ P. Attard, Phys. Rev. E 63, 61604 (2001).

${ }^{36}$ T.D.B. Jacobs, G.E. Wabiszewski, A.J. Goodman, and R.W. Carpick, Rev. Sci. Instrum. 87, 13703 (2016). 


\section{S1. Experimental details}

\section{S1.1. More details on the experimental procedure}

Experimental adhesion tests were conducted using an in situ nanoindenter (PI-95 Picoindenter, Bruker Inc., Billerica, MA) inside of a transmission electron microscope (2100F, JEOL, Tokyo, Japan). Unlike traditional nanoindentation, an AFM probe with a nanoscale apex was mounted in place of the sample and was contacted by a flat-punch diamond indenter. The AFM chip body was cleaved and glued to the sample mount of the nanoindenter. Commercial silicon AFM probes were used (PPP-NCLR, NCHR, FMR Nanosensors, Neuchatel, Switzerland), which had initial apex radii of approximately $5 \mathrm{~nm}$ and contained an oxide from the manufacturing process that extended to a height of approximately 10-15 nm at the apex. This oxide was mechanically removed by bringing the indenter into contact with the probe and sliding it in vacuum prior to testing. After removing the oxide, the silicon crystal lattice was observed to extend to the apex of the probe. Before testing, the diamond indenter was cleaned with light mechanical abrasion using a cotton swab and acetone. Next, the indenter was sequentially ultrasonicated in isopropanol, acetone, and methanol. This cleaning procedure was recommended by the manufacturer for nanoindenters, and has been used in prior studies. ${ }^{1,2}$ Further, direct inspection using the TEM verified that there was no significant debris or contamination on the surface.

\section{S1.2. In situ TEM videos of experimental contact tests}

Two videos have been included online with the supplementary material. The captions for these videos are shown below.

Video 1. A representative real-time TEM video is shown from the experimental adhesion test on a silicon probe (narrower grey shape, top) against a flat diamond indenter (wide black shape, bottom). The indenter is moved to approach the probe which causes the snap-in contact, and then the indenter is moved further resulting in the deflection of the AFM cantilever and applying a compressive maximum applied force of $17 \mathrm{nN}$. Finally, the substrate is pulled back causing the pull-off and the adhesive force of $100 \mathrm{nN}$ is measured.

Video 2. A similar representative real-time TEM video is shown from the experimental adhesion test on a different silicon probe (narrower grey shape, top) against a flat diamond indenter (wide black shape, bottom). The indenter is moved to approach the probe which causes the snap-in contact, and then the indenter is moved further resulting in the deflection of the AFM cantilever and applying a compressive maximum applied force of $305 \mathrm{nN}$. Finally, the substrate is pulled back causing the pull-off and the adhesive force of $424 \mathrm{nN}$ is measured.

\section{S1.3. Directly investigating the presence of rolling or bending of the AFM probe}

During the real-time TEM video, the probe can be directly observed. A subset of frames from one video are shown in Fig. S1. By tracing the apex of the probe when it is not in contact (Fig. S1a), this trace can be superimposed on later frames (Fig. S1b-f) to determine the instantaneous position and orientation of the probe. Because a large shape is being position-matched (rather than a single, small feature), this enables precise positioning based on matching multiple features. In our experiments, we saw no resolvable bending or rolling of the AFM probes during loading. 

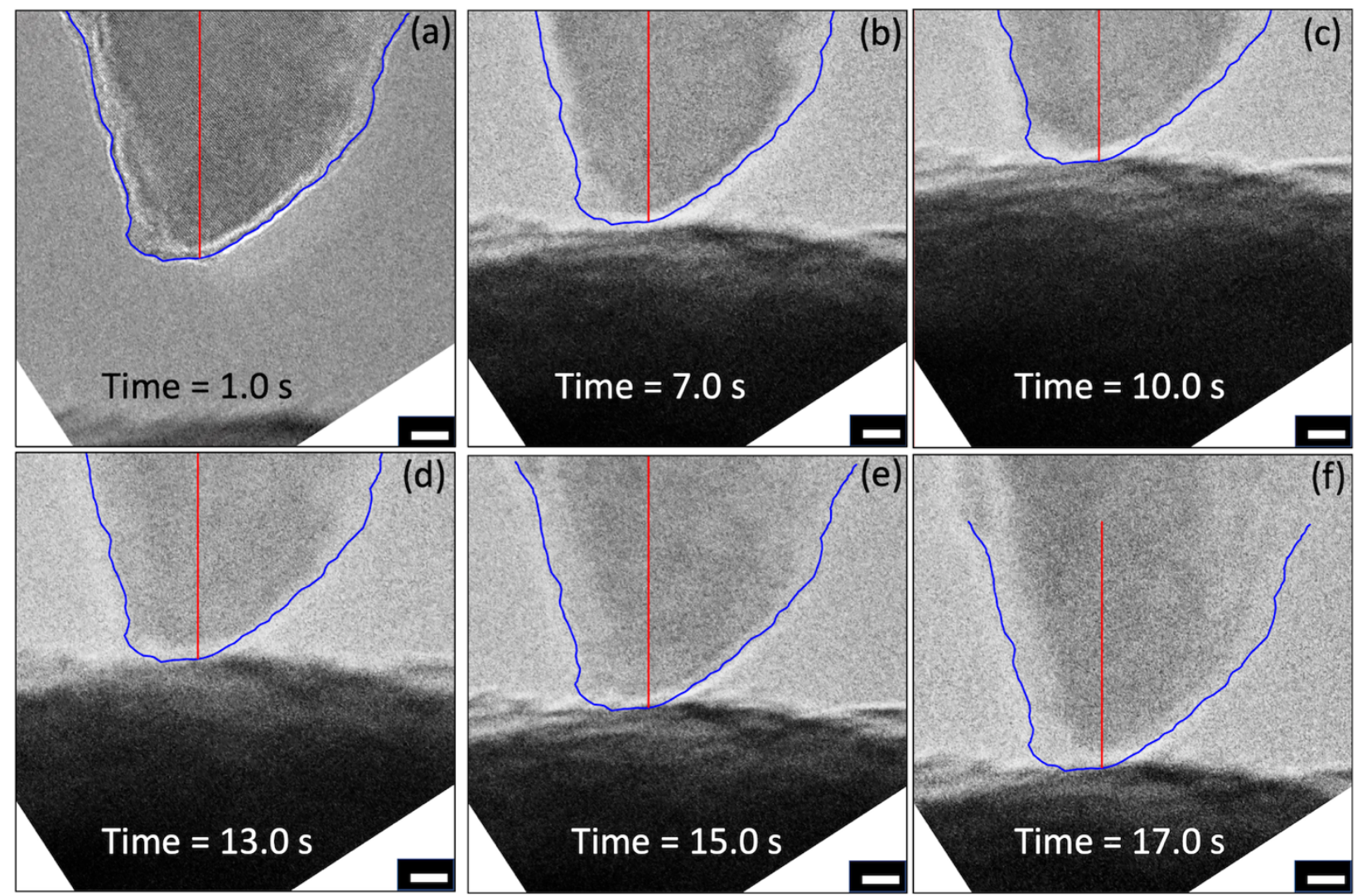

Fig. S1. The motion of the silicon probe is shown using the frames from the in situ TEM video. The outer profile of the probe (a) is traced (blue) before coming into contact, and the axis of the probe is shown by a line (red). The traced profile, together with its axis, is position-matched to the observed AFM probe in subsequent frames during loading $(\mathrm{b}, \mathrm{c})$ and unloading $(\mathrm{d}-\mathrm{f})$. This analysis indicates that there is no effect of the rolling or bending of the silicon probe under the application of the applied force. The scale bar shown in all the frames (a-f) is $5 \mathrm{~nm}$.

\section{S2. Calculation of the mean applied pressure using the elastic-plastic model}

The elastic-plastic model of Kogut and Etsion ${ }^{3}$ is used to measure the mean applied pressure at the maximum force for all the tests. This model is an extension of the Hertz model, to describe behavior after the body is predicted to exhibit local plastic deformation.

In this model, the transition from the elastic to elastic-plastic (yielding inception) occurs at the critical interference (deformation) $\omega_{c}$. At the transition, the critical pressure $p_{c}$ is calculated as $p_{c}=\frac{2}{3} K H$, where $H$ is the hardness, $K$ is a hardness coefficient that is calculated as $K=0.454+0.41 v$, and $v$ is the Poisson Ratio. For silicon, which has a Poisson ratio of 0.28 and a hardness of $13 \mathrm{GPa}^{4}$ the calculated critical pressure is $4.9 \mathrm{GPa}$.

Using the Hertz model, the normalized interference $\left(\omega / \omega_{c}\right)$ can be related to the normalized pressure $\left(p_{h} / p_{c}\right)$ $\mathrm{by}^{3}$

$$
\frac{\omega}{\omega_{c}}=\left(\frac{p_{h}}{p_{c}}\right)^{2}
$$


The mean Hertz pressure $p_{h}$ at the maximum force $F_{\max }$ for a given probe radius $R$ is given by ${ }^{5}$

$$
p_{h}=\frac{2}{3}\left(\frac{6 F_{\max } E_{\text {eff }}^{2}}{\pi^{3} R^{2}}\right)^{1 / 3}
$$

where $E_{\text {eff }}=\left[\frac{\left(1-v_{1}^{2}\right)}{E_{1}}+\frac{\left(1-v_{2}^{2}\right)}{E_{2}}\right]^{-1}$ is the effective modulus and $E$ is the elastic modulus, and subscripts 1 and 2 refer to the two materials in contact.

For $\frac{\omega}{\omega_{c}}<1$, the applied pressure is less than the critical pressure (4.9 GPa), and the contact is purely elastic. In this case, the mean pressure $p_{m}$ is given by the mean Hertz pressure (Eq. S2).

For applied pressures greater than $4.9 \mathrm{GPa}$, the contact transitions into elastic-plastic and the mean pressure is given by the following empirical equations ${ }^{3}$

For $1 \leq \frac{\omega}{\omega_{c}} \leq 6$, which corresponds to the prediction of the local yielding just below the surface with the contact being elastic, the mean pressure is given by ${ }^{3}$

$$
p_{m}=1.1075 \times p_{c} \times\left(\frac{\omega}{\omega_{c}}\right)^{0.289}
$$

For $6 \leq \frac{\omega}{\omega_{c}} \leq 110$, which corresponds to the global yielding with the contact area being elastic-plastic for $6 \leq \frac{\omega}{\omega_{c}} \leq 68$ and fully plastic for $68 \leq \frac{\omega}{\omega_{c}} \leq 110$, the prediction for the mean pressure is given by ${ }^{3}$

$$
p_{m}=1.4894 \times p_{c} \times\left(\frac{\omega}{\omega_{c}}\right)^{0.1170}
$$

\section{S3. Trends in simulated-probe results with hydrogen termination and probe size}

As discussed in the main text, the surface termination was chosen to most closely match the work of adhesion measured in the experiments, where the precise surface termination is unknown. To determine this, adhesion tests were conducted using various amounts of hydrogen surface termination from $0 \%$ to $100 \%$. The adhesion results are shown in Fig. S2. 

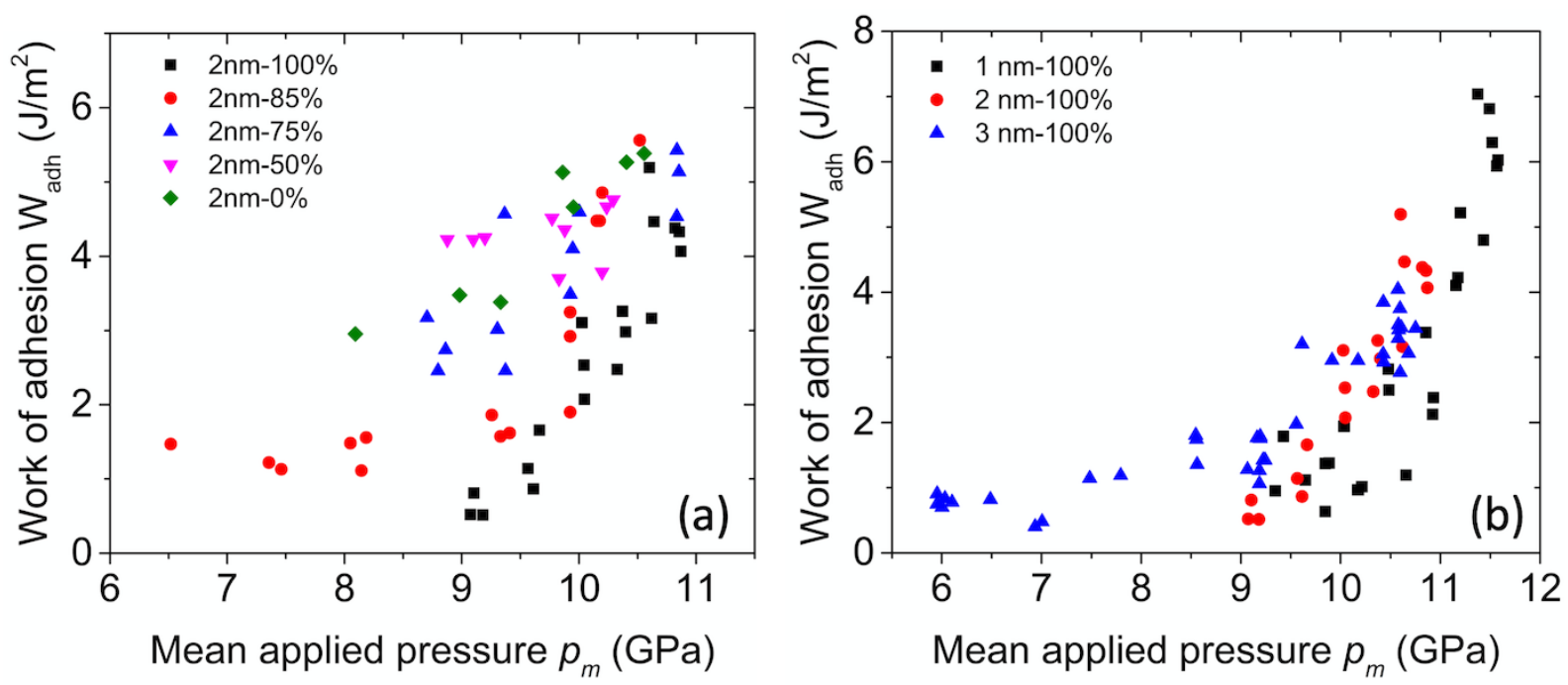

Fig. S2: The work of adhesion from the simulation varied with increasing hydrogen coverage (a), consistent with prior results, ${ }^{6}$ but data from all coverages exhibited increasing trends with applied pressure. Work of adhesion was also measured with three different probe sizes (b) with a hydrogen coverage of $100 \%$ and the trends in work of adhesion were again consistent.

\section{S4. Determining the probe radius, shape, and crystal structure from high resolution TEM}

As discussed in the main text, the shape and crystal structure was determined for all tests using highresolution imaging before and after the in situ adhesion testing. Quantitative analysis of these images were performed using custom MATLAB image-analysis routines. 

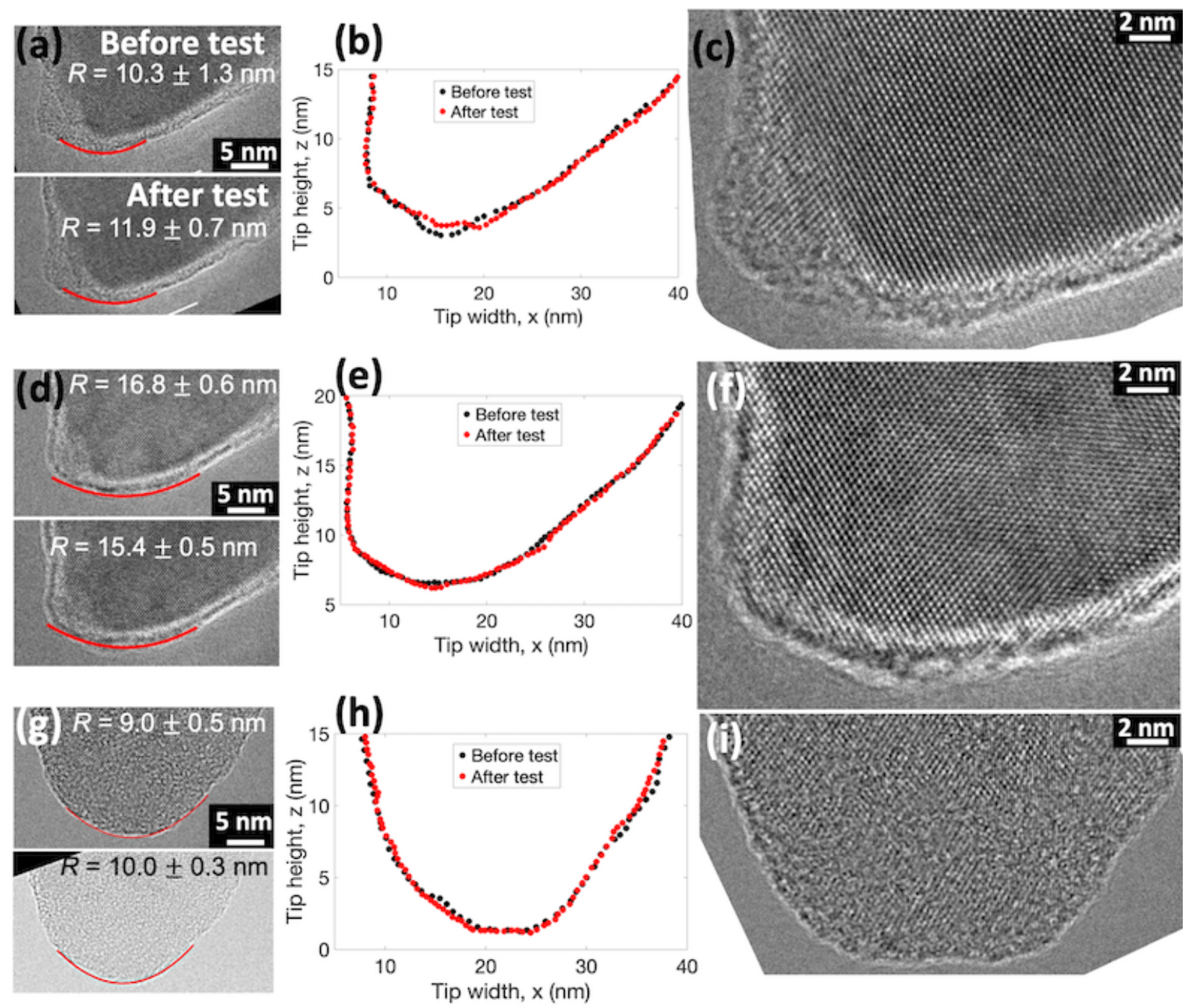

Fig. S3. High-resolution TEM images were taken before and after the test for multiple probes (Probe 1: ac, Probe 2: d-f, Probe 3: g-i) to determine probe radius, shape, and crystal structure. The TEM images were used to trace the outer profile of the probe and fit a parabola to measure the apex radius. For all the probes, there was no significant change in the radius or shape $(b, e, h)$. TEM images of the probes after the test show no evidence of the defects in the silicon lattice $(c, f, i)$. 


\section{S5. Order of testing does not affect the adhesive force}

Multiple adhesion tests were conducted on a silicon probe of radius $22.7 \mathrm{~nm}$ to measure adhesive force for different maximum applied forces (Fig. S4). Test 1 was conducted at the maximum load of $356 \pm 31 \mathrm{nN}$, which had an adhesive force of $306 \pm 31 \mathrm{nN}$. Then, test 2 was conducted at a lower maximum load of 186 $\pm 31 \mathrm{nN}$, which resulted in a smaller adhesive force of $188 \pm 31 \mathrm{nN}$. Next, tests 3,4 and 5 were conducted to the same maximum load of approximately $400 \mathrm{nN}$. These repeated tests have a similar adhesive force of approximately $430 \mathrm{nN}$. These variable and repeated measurements on the single probe suggest that the order of testing does not affect the adhesive force, and shape change by inelastic deformation plays a limited role in these contacts.

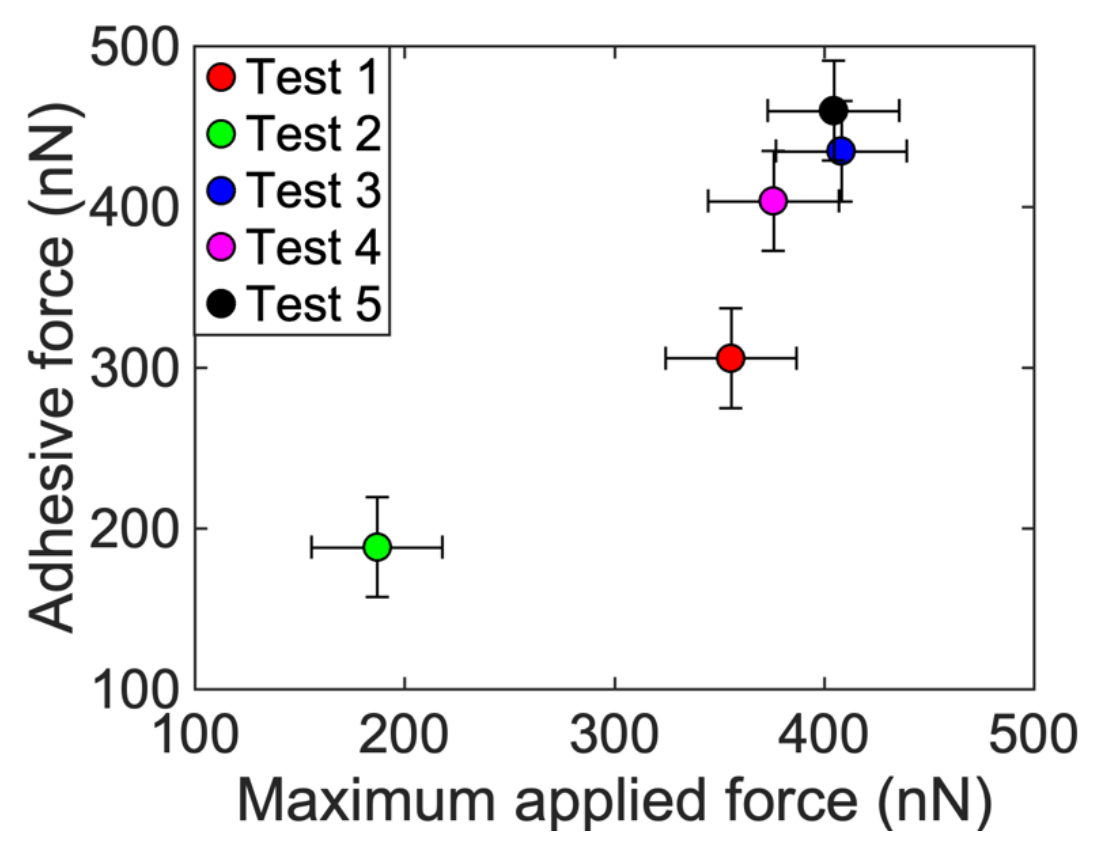

Fig. S4: Multiple adhesion tests on the silicon probe show that the order of testing does not affect the adhesive force. The increase in work of adhesion with applied pressure is not due to shape change by inelastic deformation.

\section{References}

${ }^{1}$ T.D.B. Jacobs, J.A. Lefever, and R.W. Carpick, Adv. Mater. Interfaces 2, 1400547 (2015).

${ }^{2}$ S.B. Vishnubhotla, R. Chen, S.R. Khanal, X. Hu, A. Martini, and T.D.B. Jacobs, Tribol. Lett. 67, 97 (2019).

${ }^{3}$ L. Kogut and I. Etsion, J. Appl. Mech. 69, 657 (2002).

${ }^{4}$ L.J. Vandeperre, F. Giuliani, S.J. Lloyd, and W.J. Clegg, Acta Mater. 55, 6307 (2007).

${ }^{5}$ K.L. Johnson, Contact Mechanics (Cambridge University Press, 1985).

${ }^{6}$ P.L. Piotrowski, R.J. Cannara, G. Gao, J.J. Urban, R.W. Carpick, and J.A. Harrison, J. Adhes. Sci.

Technol. 24, 2471 (2010). 